\title{
Optical links offer fast access to earth-observation sensors
}

\section{Dirk Giggenbach}

Laser terminals can boost data rates by orders of magnitude to solve the bottleneck in sensor downlinks.

Rapid or real-time access to acquired data is a challenge when operating high-resolution earth-observation (EO) sensors on board aeronautical platforms or low-earth-orbit (LEO) satellites. The transmitter can be detected and eavesdropped on easily if using radio-frequency (RF) telemetry links. In addition, standard RF links with typical data rates in the tens of megabits per second are not adequate for today's sensors. And applying for a suitably large frequency band for a mobile application, possibly for international operations, usually turns into a time-consuming nightmare. Optical downlinks through modulated laser signals provide an elegant solution. They can handle hundreds of megabits or even several gigabits per second on the optical carrier without requiring frequency authorization, thus providing a broadband and globally usable data link.

Bringing the information acquired by EO sensors to the ground is done by either landing the aeronautic platform and pulling out the mass-storage devices or RF transmission from space-based systems when the satellite comes within view of its dedicated ground station. Both methods mean delays of hours and limited amounts of transferable data. In space-based EOsystems, the downlink bottleneck limits the sensor's operational time.

Direct optical-data links provide transmission rates similar to those of terrestrial fiber. Narrow laser beams avoid interference with other systems, and the signal almost cannot be detected, intercepted, or eavesdropped. Typically, the received spot diameter is only a few tens of meters around the ground station. Transmit-antenna size can be as small as centimeters or even millimeters. The high directivity allows low transmit power in the range of a few hundred milliwatts. The receive antenna on the ground also can be quite small. Pointing to the partner is established by a combination of optical global-positioning-system and inertial-navigation-system sensors together with an agile mechanical pointing assembly. ${ }^{1}$

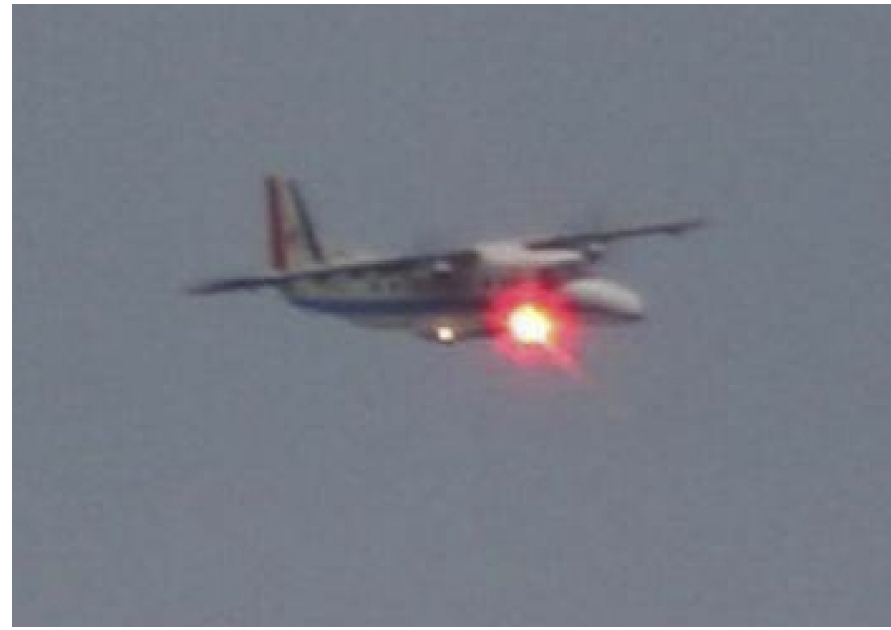

Figure 1. An airborne terminal's red visualization laser pointing towards the ground station is clearly observable at $10 \mathrm{~km}$ distance. The co-aligned $1550 \mathrm{~nm}$ data signal is not visible to the human eye.

The German Aerospace Center has performed different trials with optical-data downlink terminals from stratospheric balloons and aircraft. The latest was a fast Ethernet downlink from a Dornier-228 research aircraft (see Figures 1 and 2). We used a 1550nm laser source directly modulated with the binary data and amplified by erbium-doped fiber amplifiers to output $100 \mathrm{~mW}$ to $1 \mathrm{~W}$ power. The ground receiver was the center's optical ground station Oberpfaffenhofen (OGS-OP) in Germany. Applications for the link technology and high-resolution optical sensors include real-time traffic monitoring, monitoring of mass events, and search-and-rescue operations after natural disasters.

In the field of optical space downlinks, the center and its partners from the Japan Aerospace Exploration Agency (JAXA) and Japan's National Institute of Information and Communications Technology (NICT) have executed trials with JAXA's LEO optical interorbit communications engineering test satellite, which reliably provided an optical-data signal received by OGSOP (see Figure 3). These experiments, called the Kirari optical 


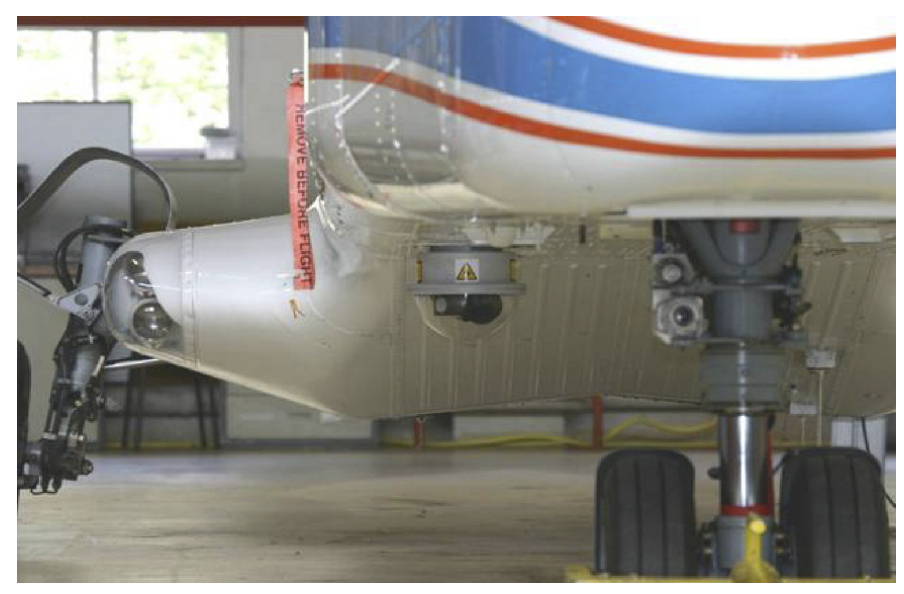

Figure 2. Aeronautical optical terminal (center of the picture), mounted underneath the German Aerospace Center's Dornier-228 research aircraft. The coarse pointing assembly is protected by a transparent dome against air drag and stone chip during takeoff and landing.

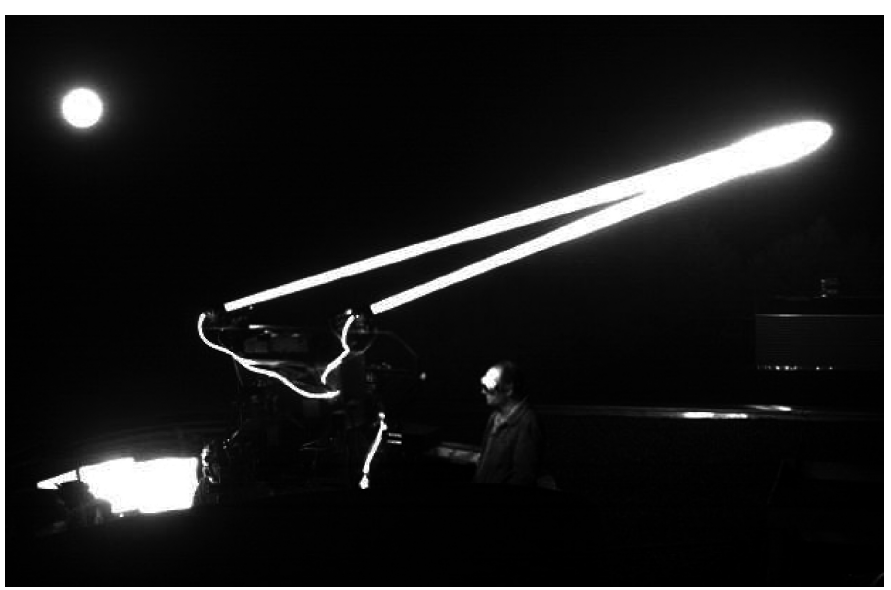

Figure 3. Optical ground station Oberpfaffenhofen (OGS-OP) during an optical satellite downlink from the Japan Aerospace Exploration Agency's optical interorbit communications engineering test satellite. Two beacon lasers are attached next to the receive telescope pointing towards the satellite to enable optical tracking. During the experiment, slight haze caused increased backscatter from the beacons and made their signal visible on this IR photograph.

downlink to Oberpfaffenhofen, proved the concept's feasibility and helped gather extensive data on the optical LEO-downlink channel. ${ }^{2}$

The technology still faces challenges. Precise tracking, especially from a fast-moving and vibrating aeronautic platform, requires sophisticated acquisition, pointing, and tracking assemblies. The index-of-refraction turbulence of the atmospheric transmission channel causes received-signal-power fluctuations that must be mitigated by error-correction coding and protocols. Link blockage by cloud cover is a minor issue in optical-sensor downlinks. Other solutions for cloud blockage are ground-station diversity and downlinks to high-altitude relay platforms. $^{3}$

In summary, to avoid the data downlink bottleneck and allow real-time access to EO-sensor data, we have implemented freespace optical-communication technology for aeronautic platforms. We have tested the technology in real-world scenarios and shown the transferability to space platforms. Further work includes terminal miniaturization, enhancement of autonomy, and securing of the data link even under strong and long fading events in mobile link scenarios.

We want to thank JAXA, NICT, and the German Aerospace Center's flight department for their collaboration in the ongoing trials. We appreciate the commitment of all members of the Optical Communications Group at the center.

\section{Author Information}

\section{Dirk Giggenbach \\ German Aerospace Center \\ Wessling, Germany}

Dirk Giggenbach heads the Optical Free-Space Communications Group. He holds a Dipl.-Ing. (MS) in electrical engineering from the Technical University of Munich and a Dr.-Ing. (PhD) from the University of the German Federal Armed Forces in Munich.

\section{References}

1. J. Horwath and C. Fuchs, Aircraft to ground unidirectional laser-communication terminal for high-resolution sensors, Proc. SPIE 7199, p. 719909, 2009. doi: $10.1117 / 12.808869$

2. Y. Takayama, T. Jono, M. Toyoshima, H. Kunimori, D. Giggenbach, N. Perlot, M. Knapek, K. Shiratama, J. Abe, and K. Arai, Tracking and pointing characteristics of OICETS optical terminal in communication demonstrations with ground stations, Proc. SPIE 6457A, p. 645707, 2007. doi:10.1117/12.708417

3. D. Giggenbach, B. Epple, J. Horwath, and F. Moll, Optical satellite downlinks to optical ground stations and high-altitude platforms in: Advances in mobile and wireless communications - views of the 16th IST mobile and wireless communication summit, Lect. Notes Electr. Eng. 16, Springer, 2008. ISBN: 978-3-540-79040-2 